\title{
Bending-wave localization and interaction band gaps in quasiperiodic beams
}

\author{
B. Van Damme $\odot,{ }^{*}$ L. Matar, G. Hannema, D. Tallarico $\odot$, A. Zemp $\odot$, and A. Bergamini \\ Laboratory for Acoustics/Noise Control Empa, Materials Science and Technology \\ and Ueberlandstrasse 129, 8600 Duebendorf, Switzerland
}

(Received 22 December 2020; accepted 8 February 2021; published 1 March 2021)

\begin{abstract}
Aperiodic metamaterials can host topological or localized wave modes, and gradually changing structures are known to induce conversion between elastic wave types. This work describes a family of one dimensional, aperiodic elastic structures that can host localized modes or achieve low-frequency attenuation by varying a single geometrical parameter, i.e., the ratio between the sizes of two interacting periodic structures. Two periodic sets of slits on the top and bottom of a slender beam scatter bending waves. The thickness profile and cross section of beams with a period ratio close to 1 varies slowly, resulting in localized wave modes. The localization happens because of spatially confined band gaps and is only triggered for certain excitation locations, unlike wave localization due to a local defect. If the periods differ more, broad band gaps appear at wave numbers equal to linear combinations of the individual Brillouin zone edges of each set of slits. We call these interaction band gaps, since they result from the interplay between two periodic sets of slits. Low-frequency wave attenuation is achieved while maintaining a high overall bending stiffness.
\end{abstract}

DOI: 10.1103/PhysRevB.103.094301

\section{INTRODUCTION}

Metamaterial research, be it in optics, acoustics, or dynamics, mainly focuses on periodic structures. This is evident for photonic and phononic crystals, whose functionality depends on wave interaction due to Bragg scattering [1]. The advantage of unit cell modeling, which allows the prediction of the dispersion relation with minimal calculation effort [2], is often also exploited for locally resonant metamaterials [3]. Their efficiency, however, does not rely on a periodic placement of the individual resonators, as long as they are placed sufficiently close to each other [4]. In recent years, aperiodic metastructures have been receiving increasing attention. Explicit models of large structures are in reach thanks to high-performance computing. This allows the assessment of boundary effects in finite metamaterials, or the effect of defects in a periodic pattern. Aperiodic structures demonstrate various interesting properties that cannot exist in periodic arrays. A well-studied family of aperiodic metamaterials consists of the assembly of two periodic structures. The sudden jump in properties at the interface introduces a plethora of effects, such as wave localization $[5,6]$, topologically protected waves [7-9], and various quantum-like elastic waves [10-12]. Another class of aperiodic metastructures has a gradually varying profile. Metawedges, for example, consist of an array of slowly varying resonators that can lead to wideband "rainbow" trapping or mode conversion, depending on the direction of incidence of the waves $[13,14]$. Thickness or stiffness modulation of beams and plates also leads to gradual, adiabatic changes with topological wave effects $[15,16]$. Alternatively, long-range periodicity can be broken in a controlled way using the concept of quasicrystals [17-19]. Examples are known in

*bart.vandamme@empa.ch one, two, or three dimensions, e.g., Fibonacci sequences [20], modulation of a periodic sequence $[15,21,22]$, or certain tessellations [23]. Quasicrystals exhibit no translational periodicity, but they contain local symmetries interacting with impinging wave fields [24].

The topic of this article is a family of one-dimensional quasiperiodic metamaterials, achieved by superposing two periodic arrays of slits on the top and bottom of an elastic beam to scatter bending waves. The periodic arrays are chosen in such a way that no periodicity is present within a finite sample of $1 \mathrm{~m}$ length. The characteristics of the overall wave propagation in these quasiperiodic beams are not a result of Bragg scattering, which occurs in periodic lattices, but they are affected by the interaction of both sets of scattering slits. After introducing the geometry of the investigated aperiodic beams, the effect of the slits on wave propagation is qualitatively described by an inquiry of the reciprocal space. The following three sections address bending wave scattering in three distinct cases: a periodic beam with a local defect, a slowly varying aperiodic beam, and a quickly varying aperiodic beam. These cases are investigated numerically, using finite-element models, after which the effects are validated experimentally.

\section{QUASIPERIODIC BEAMS WITH SUPERIMPOSED SCATTERING SLITS}

In a beam with thickness $T$, two sets of rectangular slits are milled from the top and bottom surface, with a depth $d<T / 2$. Each set of slits is periodic with period $p_{1}$ and $p_{2}$, respectively. Mathematically, the profile can be derived from a pulse wave with duty cycle $a$ and period $p$,

$$
\Pi_{a}\left(\frac{2 \pi}{p} x\right)=\Pi_{a}(k x)= \begin{cases}1 & \text { if } x \bmod p<a \\ 0 & \text { if } x \bmod p>a .\end{cases}
$$


(a)

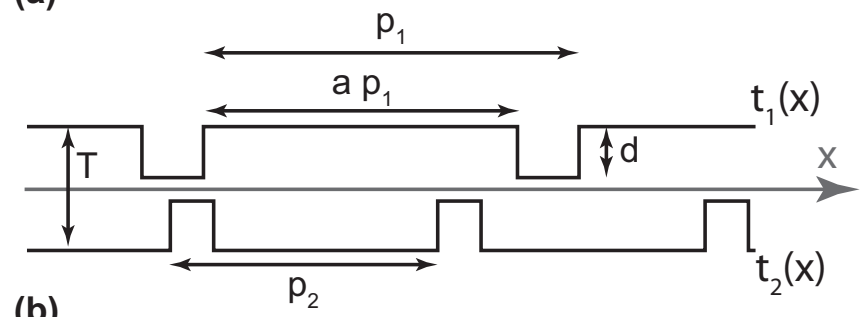

(b)

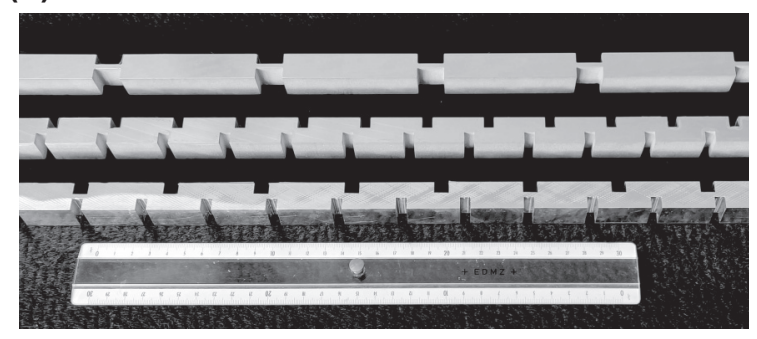

FIG. 1. Schematic presentation of the beam geometry, with two periodic sets of slits (a). Experimental samples with period ratios, from top to bottom, $r_{1}=p_{1} / p_{2}=1, r_{2}=1.028$, and $r_{3}=1.405$ (b).

The top $(i=1)$ and bottom $(i=2)$ profile of the beam can now be written as

$$
t_{i}(x)=(-1)^{(i+1)}\left(d_{i} \Pi_{a_{i}}\left(k_{i} x+\phi_{i}\right)+\frac{T-2 d}{2}\right) .
$$

The thickness profile of the beam is given by

$$
h(x)=t_{1}(x)-t_{2}(x)
$$

and can take four distinct values: $T, T-d_{1}, T-d_{2}$, and $T-$ $d_{1}-d_{2}$. In this paper, the slit depths and duty cycles of the top and bottom profile are chosen to be identical, and the phase shift $\phi_{1}=\phi_{2}=0$ so that each beam starts with a whole thick section at $x=0$. The quasiperiodic beam is thus fully defined by the period ratio $r=p_{1} / p_{2}$.

If the ratio of the periodicities $r$ is rational, say equal to the irreducible fraction $r=m / n$ with $m, n \in \mathbb{N}$, a periodic supercell can be identified with length $p_{s}=n p_{1}=m p_{2}$. Incommensurate sets of scatterers are defined by an irrational value of $r$, in which case no supercell exists. The value of $r$ can nevertheless always be approximated by a rational number. However, in either case the values of $m$ and $n$ can be so high that the supercell is larger than the beam's length and no periodicity whatsoever occurs within a sample of realistic size.

In this article, three distinct cases are identified depending on the value of $r$, as shown in Fig. 1(b). The first is the wellstudied periodic beam with $r_{1}=1$, with perfect alignment of the slits. All thin sections coincide leading to a low bending stiffness and therefore band gaps at relatively low frequencies. Beam 2 is defined by a ratio slightly larger than $1, r_{2}=1.028$, which leads to a slow variation of the thickness profile. The thick sections at the start of the beam are aligned, and they remain almost aligned for several slits. Locally, within a range of up to five slits, the beam can be assumed to be periodic. On a larger length scale, however, the relative position of the top and bottom slit varies from perfectly aligned to completely out of phase. Beam 3 has a periodicity ratio $r_{3}=1.405$, leading
TABLE I. Geometry parameters of the three beams.

\begin{tabular}{lccc}
\hline \hline Beam & $r$ & $p_{1}(\mathrm{~mm})$ & $p_{2}(\mathrm{~mm})$ \\
\hline 1 & 1.000 & 97.9 & 97.9 \\
2 & 1.028 & 37.0 & 36.0 \\
3 & 1.405 & 51.6 & 36.7 \\
\hline \hline
\end{tabular}

to a quick variation in the thickness profile so that no local periodicity can be found. In this case, a longer length scale shows a certain translational symmetry: $r_{3}$ can be approximated as $7 / 5$, and sections with length $5 p_{1}=7 p_{2}$ are almost identical. The slit periodicity $p_{2}=36 \mathrm{~mm}$ is chosen so that both quasiperiodic beams 2 and 3 would require excessive lengths to form an actual supercell: $9.2 \mathrm{~m}$ for beam 2 and $10.3 \mathrm{~m}$ for beam 3 , so that no periodicity can arise within the chosen sample lengths of $1 \mathrm{~m}$. The unit cell of beam 1 is optimized to have a first band gap at the same frequency as beam 3, as will be shown later in the paper. An overview of the parameters defining the beams' geometries is given in Table I.

For practical purposes, the beams in this study are made of aluminum. Both arrays of slits have the same depth, resulting in asymmetric beams as illustrated in Fig. 1(a) with thick sections $(T=17 \mathrm{~mm})$, thin sections $(t=3 \mathrm{~mm}$, resulting from $7 \mathrm{~mm}$ deep slits on either side), and medium sections $(10 \mathrm{~mm})$. The slit width is chosen as $1 / 6$ of the respective periods $p_{1}$ and $p_{2}$ in order to maintain a constant mass-per-length for each choice of the period ratio $r=p_{1} / p_{2}$. The only parameter affected by the quasiperiodicity is the overall static bending stiffness of the beam. In beams with a significant portion of thin sections, in particular beam 1, the static deflection is evidently much higher than in the quasiperiodic beams. To quantify this effect, the deflection of the beams under a $1 \mathrm{~N}$ load at the right end, while keeping the left end clamped, was modeled in Ansys 19.2. The total deflection was $25.14 \mathrm{~mm}$ for beam $1,9.88 \mathrm{~mm}$ for beam 2 , and $5.24 \mathrm{~mm}$ for beam 3 . The average static bending stiffness is five times higher in the last case compared to the periodic beam.

\section{DISPERSION AND WAVE PROPAGATION IN BEAMS WITH RANDOM THICKNESS PROFILES}

In periodic beams, Bloch-Floquet boundary conditions can be applied on a unit cell to predict bending wave dispersion [2]. This technique can to a certain extent be used for quasiperiodic structures, if the sample is large compared to a supercell. This is the approach of [15], to prove the existence of localized wave modes in the presence of point scatterers with modulated periodicity. The goal of this article, however, is to investigate the influence of the quasiperiodicity parameter $r$ on the dynamic response of beams much shorter than a supercell. Under these circumstances, the theoretical dispersion relation for infinite samples is not a viable analysis approach. Moreover, the scatterers under consideration are not pointlike (masses, springs, or resonators), but they have a finite extent. This increases the modeling complexity, since no analytical methods can be used as for point scatterers [25]. 
Bragg scattering in (phononic) crystals arises when the wave number $k$ coincides with the reciprocal lattice of the waveguide. Flexural waves in thin beams obey the EulerBernoulli wave equation

$$
\frac{\partial^{2}}{\partial x^{2}}\left(E I \frac{\partial^{2} w}{\partial x^{2}}\right)+\rho A \frac{\partial^{2} w}{\partial t^{2}}=0
$$

where $E$ is Young's modulus, $I=b h^{3} / 12$ is the second moment of area for a rectangular beam cross section with width $b$ and thickness $h, \rho$ is the mass density, and $A=b h$ is the cross-sectional area. In homogeneous thin beams made out of one single material, the wave number is solely defined by the square of its thickness. Therefore, we assume that for beams with varying thickness $h(x)$, the lattice responsible for wave scattering is defined by the function $h(x)^{2}$. The reciprocal lattice $H(k)$ can be approximated by the Fourier transform of the squared values of Eq. (3):

$$
H(k)=\mathscr{F} h(x)^{2}=\mathscr{F}\left(t_{1}^{2}+t_{2}^{2}-2 t_{1} t_{2}\right) .
$$

The Fourier series of a rectangular function $\Pi_{a}\left(k_{i} x\right)$ takes nonzero values at $k=n k_{i}, n$ being a whole number. Since $t_{1}(x)^{2}$ and $t_{2}(x)^{2}$ are in turn rectangular functions, the Fourier series of the thickness profile in this case consists of nonzero values at $k_{1}, k_{2}$, and their linear combinations $a k_{1}+b k_{2}, a$ and $b$ being positive or negative whole numbers. The latter wave numbers arise from the convolution of the reciprocal lattices of the top and bottom beam profiles $T_{1}(k) * T_{2}(k)$. This nonlinear interaction between the two sets of slits introduces new high-symmetry points in reciprocal space, and thus Bragg scattering at wave numbers differing from $k_{1}$ and $k_{2}$. The additional scattering points will result in more band gaps, which we call interaction band gaps.

The reciprocal lattice strongly depends on the period ratio $r$ and duty cycle $a$, as shown in Figs. 2(a) and 2(b). The graphs were calculated as the fast Fourier transform of a finite beam of $2 \mathrm{~m}$. If $r$ is close to whole values, only the Brillouin zones of the independent slit arrays can be distinguished. At fractions leading to short supercells, e.g., $3 / 2$ or $4 / 3$, one or two additional wave numbers can be found within the first Brillouin zone. Ratios leading to long supercells can lead to four or five additional interaction scattering wave numbers in the first Brillouin zone. Values around $r=1.4$ and 1.6 show several distinct peaks below $40 \mathrm{rad} / \mathrm{m}$. The prominence of these scattering wave numbers depends on the duty cycle as well. Panel (b) shows that three out of four peaks associated with $r=\sqrt{2}$ disappear at $a=0.5$. The value $a=5 / 6$ leads to four more or less equally prominent interaction scatterers within the smallest Brillouin zone. The phase shift between the two sets of slits does not play a significant role in the reciprocal lattice, as shown in panel (c). It will be shown in Sec. V, however, that the dynamic response of the finite beam depends on the excitation location, and thus on the local phase between the two sets of scatterers.

The choice of the three prototypes described in the previous section follows from this analysis. The choice for $r=$ 1.028 is sufficiently close to $r=1$ so that no interaction scattering wave numbers can be distinguished. The case $r=$ 1.405 should show a maximum amount of interaction band gaps. Their reciprocal lattices are shown in Fig. 5(c).
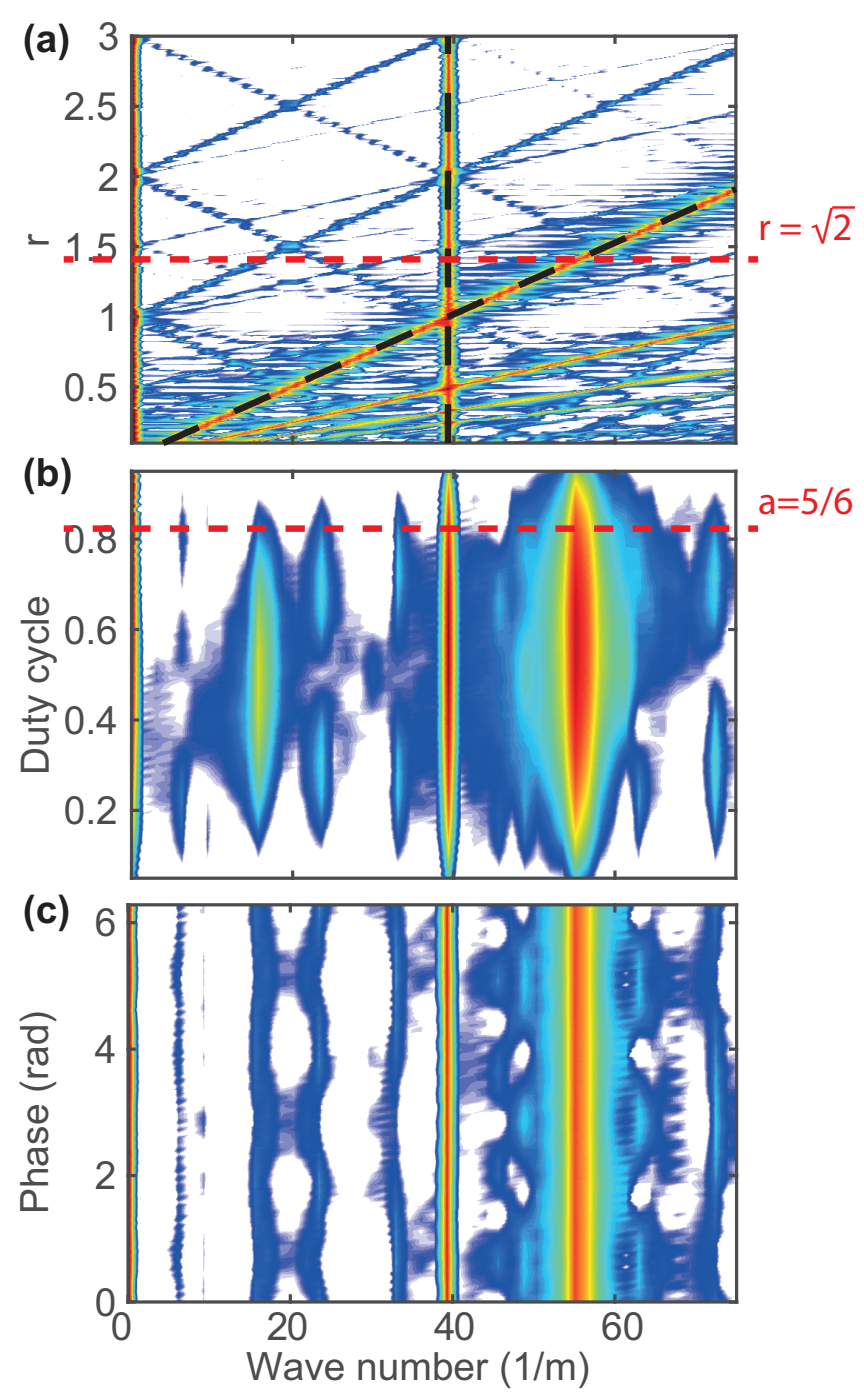

FIG. 2. Reciprocal lattice of beams with a double array of slits, as a function of (a) period ratio with duty cycle $a=5 / 6$, (b) duty cycle for $r=\sqrt{2}$, and (c) phase shift of the bottom array with respect to the top array for $r=\sqrt{2}$ and $a=5 / 6$. Panel (a) shows the edge of the first Brillouin zone of both arrays as black dashed lines. The full thickness of the beam is $T=17 \mathrm{~mm}$, the slit depth is $d=7 \mathrm{~mm}$.

The frequency-wave number relation of waves propagating in any random sample can be calculated using the frequency response function (FRF) along a straight line of points [26]. The most straightforward way is to calculate the (spatial) fast Fourier transform (FFT) of the complex FRF $s\left(x, f_{m}\right)$ measured along the beam at each frequency $f_{m}$. The spatial FFT for each frequency line is normalized to a maximum value of 1 in order to reduce the effect of the frequency-dependent amplitude of the waves. Since the FFT method only provides the real part of the wave number, the inhomogeneous wave correlation (IWC) method can alternatively be used to assess the imaginary part [26]. This method optimizes the correlation between the measured profile $s\left(x, f_{m}\right)$ with an inhomogeneous damped propagated wave $\hat{o}=\exp (i(k+i g) x)$, with $k$ and $g$ being the real and imaginary part of the wave number, respectively. This has to be repeated for all frequencies $f_{0}$, in which 


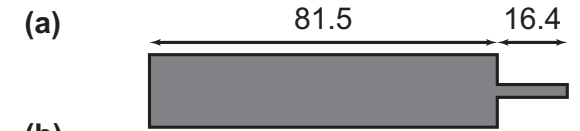

(b)
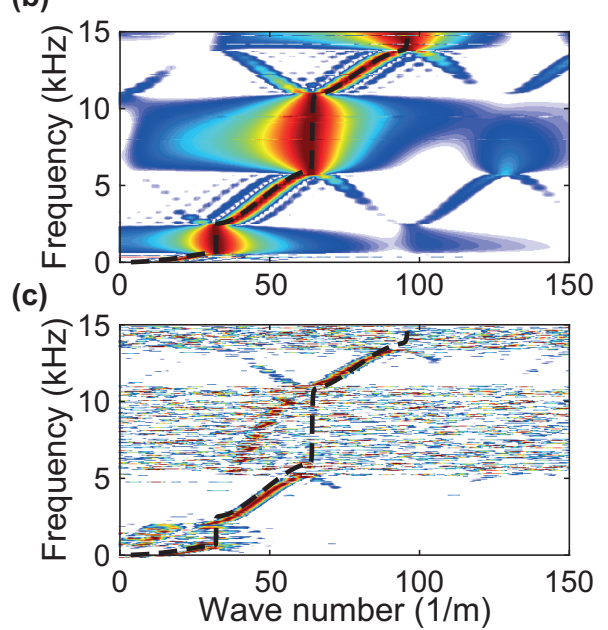

(d)

(e)

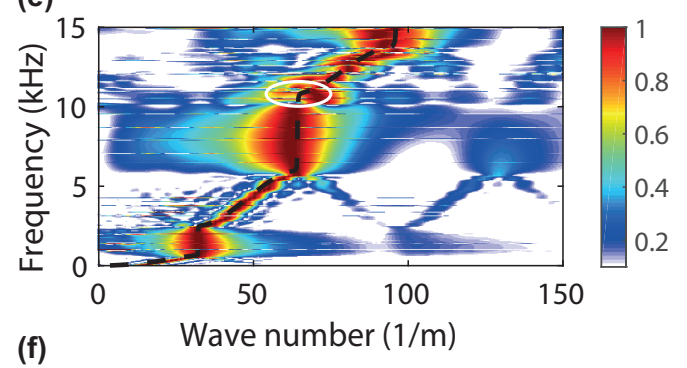

(f)

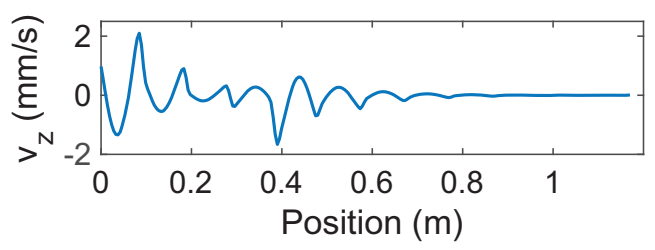

FIG. 3. Benchmark periodic sample with $p_{1}=p_{2}=97.9 \mathrm{~mm}$ (a). Dispersion relation retrieved from a finite-element simulation (b) and experimentally (c). The black dashed curve is the analytically predicted dispersion. Mode conversion from flexural to longitudinal waves can be clearly seen in the second band gap of the experimental results. The effect of a localized mass perturbation (d) is visible as sharp perturbations of the pristine beam above $10 \mathrm{kHz}$ (e). An example of a localized mode with high velocity amplitude around the mass perturbation for $f=10.2 \mathrm{kHz}$ is shown in (f).

case the maximum of the function

$$
\operatorname{IWC}(k, g)=\frac{\left|\int s\left(x, f_{0}\right) \cdot \hat{o}^{*}(x, k, g) d x\right|}{\sqrt{\int\left|s\left(x, f_{0}\right)\right|^{2} d x \cdot \int|\hat{o}(x, k, g)|^{2} d x}}
$$

is the best match between the measured and predicted attenuated wave.

For this work, the FRFs along the three beams are retrieved both numerically and experimentally. The beams are excited at one side under free-free boundary conditions, thereby avoiding excitation in a vibration node. The numerical model of the harmonic response was created in Ansys 19.2, with 2000 frequency steps from 50 to $15000 \mathrm{~Hz}$. The mesh contains hexahedral elements with a maximum size of $5 \mathrm{~mm}$. The predicted maximum wave number for each geometry at $15 \mathrm{kHz}$ is approximately $100 \mathrm{~m}^{-1}$, meaning a minimum wavelength of $31 \mathrm{~mm}$. The choice of the element size ensures at least six elements per wavelength along the propagation direction, which is sufficient to avoid numerical dispersion effects. The material properties were defined as $\rho=2770 \mathrm{~kg} / \mathrm{m}^{3}$ for the mass density and $71 \mathrm{GPa}$ for Young's modulus. The harmonic analysis uses a $1 \mathrm{~N}$ force on the left edge, and the right edge can move freely. The complex frequency response function was exported for all nodes along the central axis.

The FRF of the three beams, placed on two soft pads to approximate free boundary conditions, was measured using the velocity response to a dynamic force input. A shaker excited one end of the beam with a linearly swept sine signal $(50-15000 \mathrm{~Hz})$, using a PCB $208 \mathrm{C} 01$ sensor to measure the force. The out-of-plane velocity was measured by a Polytec PSV-400 scanning vibrometer in $10 \mathrm{~mm}$ steps along the length of the beam. This is in accordance with the Nyquist sampling criterion, which requires a measurement in points maximum half the smallest wavelength apart. The Polytec system was used to generate the excitation signal and acquire the force sensor data, and to calculate the H1 FRF estimation (velocity/force). The data acquisition was set to a bandwidth of $20 \mathrm{kHz}$, measuring $102400 \mathrm{FFT}$ lines in a $5.12 \mathrm{~s}$ signal. The measurement was repeated six times in each point to reduce the noise of the FRF.

\section{WAVE LOCALIZATION IN A PERIODIC BEAM WITH MASS DEFECT}

The method described in the previous section to calculate the dispersion in finite beams is validated by the analytical results for a periodic beam, as shown in Figs. 3(b) and 3(c). For the unit cell in Fig. 3(a), the dispersion relation of an infinite beam can be calculated using the transfer-matrix method as described in [27]. The resulting black dashed lines overlap with results of the numerical and experimental data, showing that the method is accurate for samples of this size, containing 12 periods.

A single local defect or imperfection in a perfectly periodic sample always leads to energy accumulation and therefore local modes at the defect location [28]. Such modes are not topologically protected, and slightly changing the defect itself or the surrounding material leads to completely different results. They are mostly unwanted, since they can lead to high amplitudes in a confined area, as seen in Fig. 3(f). The effect of the band gap, leading to decreasing amplitude away from the source at the beam's left edge, is annihilated close to the mass perturbation at $x=0.4 \mathrm{~m}$. 

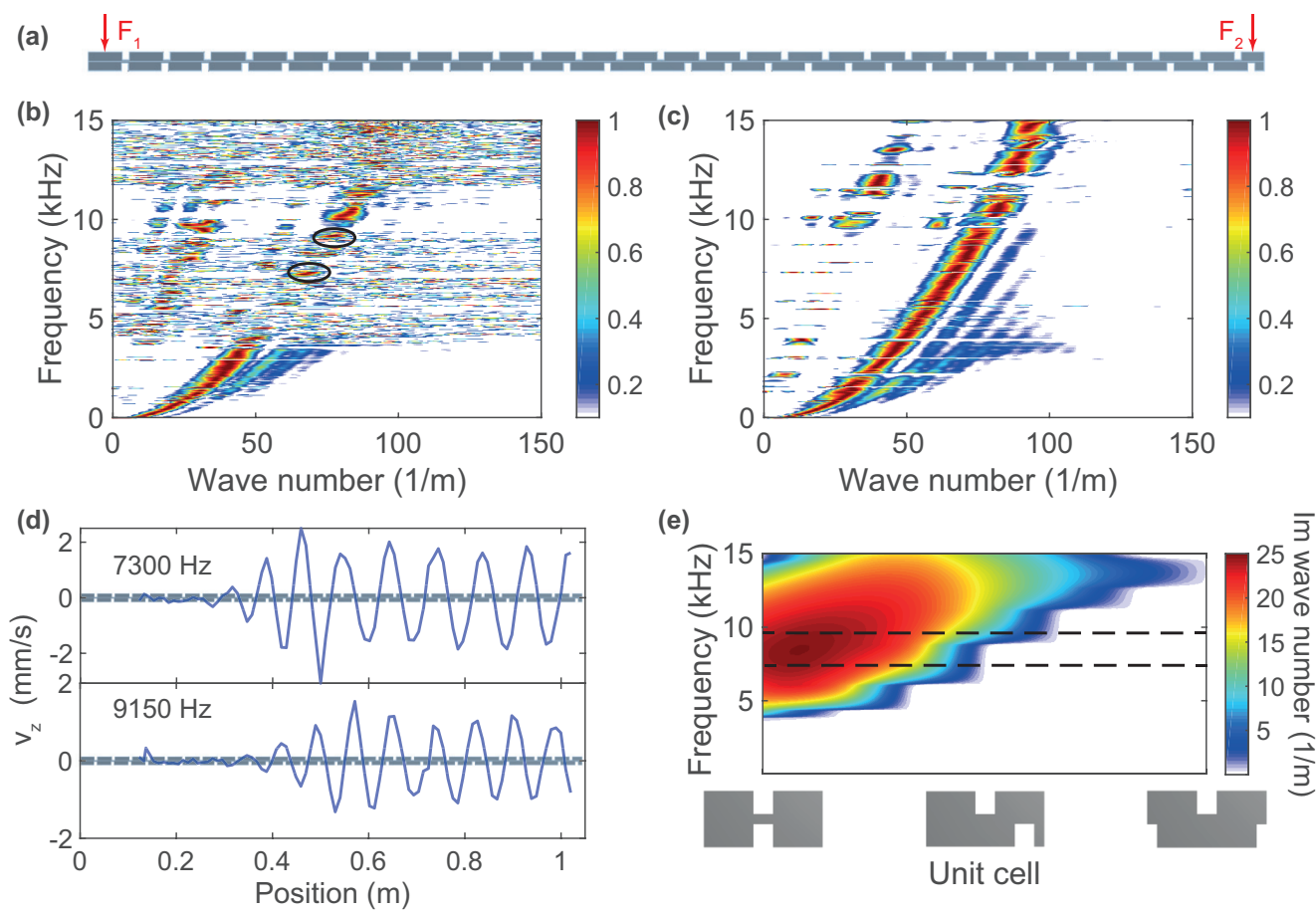

FIG. 4. Adiabatically varying beam geometry (a) with $r=1.028$ and $p_{1}=37.0 \mathrm{~mm}$. Experimentally measured wave number-frequency relationship for excitation point $F_{1}$ (b) and $F_{2}$ (c). Two localized modes measured for excitation point $F_{1}(\mathrm{~d})$, at frequencies highlighted in (b). Band-gap efficiency as a function of the location of the bottom slit, assuming periodicity (e). The black dashed lines show the frequencies of the localized modes in (d). At $9150 \mathrm{~Hz}$, the band gap extends further into the beam, explaining why the localized mode appears $100 \mathrm{~mm}$ further compared to the $7300 \mathrm{~Hz}$ mode.

\section{WAVE LOCALIZATION IN ADIABATIC BEAMS}

Varying the period ratio $r$ does not cause local defects like a point mass, but the bending wave propagation is affected by a gradually changing thickness profile. As described in [16], the phase between the slit patterns changes slowly, leading to topological effects. In this case, the beam geometry varies slowly if $r$ is close to 1 , and a random section with length $p_{1} \simeq$ $p_{2}$ is almost identical to the neighboring sections. Such slow variations are also referred to as adiabatic [29]. In reciprocal space, the interaction wave numbers cannot be discerned, as can be seen in Fig. 2(a). The high-symmetry wave numbers are slightly smeared out compared to the perfectly periodic beam.

Beam 2 is an example of this case, with $p_{1}=37.0 \mathrm{~mm}$ and $r=1.028$ as presented in Fig. 4(a). Since the beam can be considered periodic at each location, the imaginary part of the Bloch wave number can be calculated using the transfermatrix method [27]. The variation of the band-gap location and depth is shown in Fig. 4(e) for various locations of the bottom slit. The first band gap gradually shifts to higher frequencies if the slit shifts from perfect alignment with the top to its extremal location in the middle between top slits. This is similar to the rainbow trapping mechanism of metawedges, where gradually changing local resonators are placed on a wave guide. Harmonic waves can propagate up to the point where the band gap for their particular frequency opens. This is made clear from the wave number-frequency diagrams measured for two excitation points, $F_{1}$ and $F_{2}$ in Fig. 4(a). The geometry close to point $F_{1}$ has a band-gap opening below
$5 \mathrm{kHz}$, leading to a noisy signal and mode conversion in the approximated dispersion diagram [Fig. 4(b)]. If, on the other hand, the beam is excited in $F_{2}$, waves do not immediately encounter a band gap below $15 \mathrm{kHz}$ [Fig. 4(c)]. They can freely propagate over a large section of the beam, with an almost ideal parabolic dispersion. Wave propagation is expected to be symmetric in infinitely long beams, since there is no disturbance of the time parity or space-time modulation [30]. The perceived difference of dynamic responses of the same beam is purely an effect of the boundary conditions, especially the location of the excitation force.

As an effect of the wide band gap for an excitation in point $F_{1}$, several localized modes can be identified. Above $5 \mathrm{kHz}$, some sharply defined frequency excitations lead to dispersion points along the standard parabola. Two of them are highlighted in Fig. 4(b), and the measured velocity profile along the beam is shown in Fig. 4(d). Although the beam is excited at frequencies generating bending waves that are highly attenuated close to the source, sufficient energy is pumped into the remainder of the beam to build up a localized mode. The higher frequency mode starts farther right, in accordance with the band-gap efficiency for that section, as shown by the dashed black lines in Fig. 4(e).

\section{INTERACTION BAND GAPS IN BEAMS WITH A QUICKLY VARYING THICKNESS PROFILE}

So far, the predicted and measured effects of quasiperiodic beams are in accordance with known results in metawedges and space-modulated point scatterers. If, however, the period 
(a)

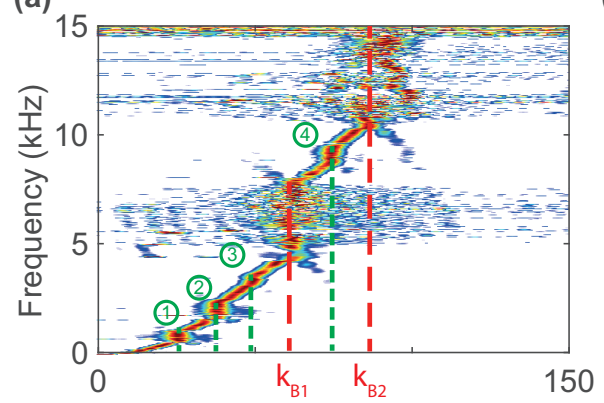

(c)

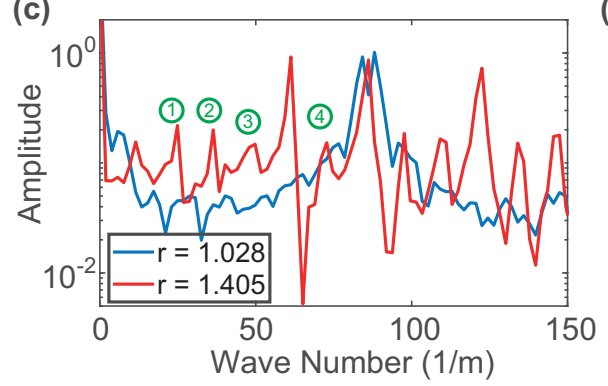

(b)

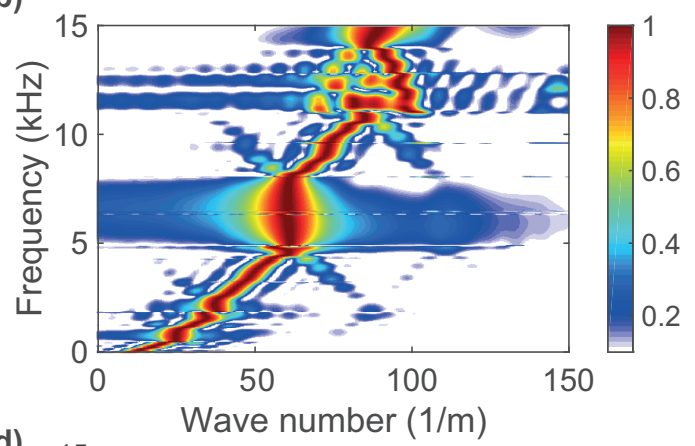

(d)

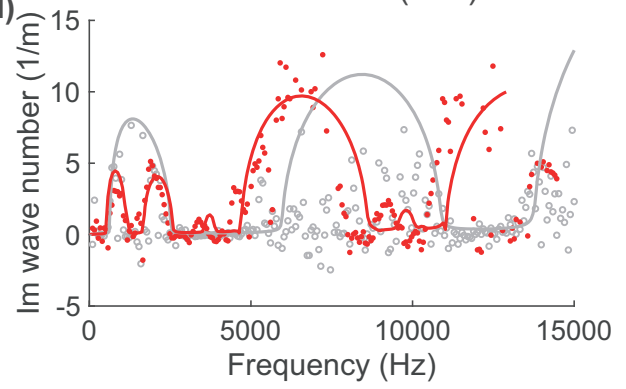

FIG. 5. Measured (a) and modeled (b) wave number-frequency relation of an aperiodic beam with $r=1.405$ and $p_{1}=51.5$ mm, showing interaction band gaps (1), (2), (3), (4). Fourier transform of the thickness function $h^{2}$ of beams 2 and 3, showing exposed peaks at the location of the interaction band gaps for a rapidly varying beam (c). Imaginary part of the wave number measured by the inhomogeneous wave correlation method for a periodic and rapidly varying beam (d). Dots show measurements of beam 1 (gray) and beam 3 (red); solid lines are the corresponding model results.

ratio gets larger, an interesting effect becomes evident in reciprocal space. Several interaction wave numbers can be clearly distinguished, all pointing out possible high-scattering points for bending waves. To illustrate this, beam 3 has a period ratio $r=1.405$ and $p_{1}=51.6 \mathrm{~mm}$. The Fourier transform of $h(x)^{-1 / 2}$ is shown in Fig. 5(b). Two high peaks are visible at the Brillouin zone edges $k_{B 1}=\pi / p_{1}$ and $k_{B 2}=$ $\pi / p_{2}$. Due to the quickly varying thickness function $h(x)$, interaction peaks appear at linear combinations of these two values $k_{i}=a k_{B 1}+b k_{B 2}$, with $a, b \in \mathbb{Z}$. Since $a$ and $b$ can be negative, additional band gaps appear at wave-number values below the first Brillouin zone $k_{B 1}$, shown as the peaks (1) ( $a=$ $-1, b=1)$, (2) $(a=2, b=-1)$, and (3) $(a=-2, b=2)$. The interaction band gaps are clearly visible in the approximated dispersion diagram of Fig. 5(a).

The fact that no interaction band gaps are seen in the slowly varying beam 2 can be appreciated from its Fourier decomposition as well [Fig. 5(b), blue curve]. Both Brillouin zone edges merge into one broad peak, but no exposed peaks appear at interaction wave numbers. This puts a limitation on the achievement of low-frequency band gaps. It might seem logical that two slightly different periodic slit arrays would result in a very low-frequency band gap due to the small value of $k_{B 2}-k_{B 1}$. Our results, however, show that individual interaction peaks must be discernible in the beam's $h(x)^{2}$ spectrum.

Based on the numerical model, the value of $p_{1}$ was optimized so that the first large interaction band gap starts at the same frequency as the first band gap of periodic beam 1. The question remains if the interaction band gaps of the quasiperiodic beam are as efficient as the band gaps of a periodic beam. To assess this, the imaginary part of the wave number was measured using the IWC method. The results for beam 1 and beam 3 are shown in Fig. 5(c). The aperiodic beam 3 can be approximated by a beam with $r=1.4$, resulting in a supercell with length $5 p_{1}=7 p_{2}=258 \mathrm{~mm}$. Applying periodic boundary conditions on the unit cell of beam 1 and the supercell of beam 3 according to [2] results in the solid red and gray lines in Fig. 5(d).

The low-frequency band gaps appear to have an additional advantage over the periodic beam. The measured values for the periodic beam 1 are much affected by mode conversion, and they do not reach the predicted high values from the transfer-matrix method. This was already described in [31]. However, the interaction band gaps are narrow enough not to suffer much from mode conversion, and the typical band-gap pattern for phononic crystals can be measured for frequencies up to $15 \mathrm{kHz}$. The difference between model and measurements at higher frequencies is due to the approximation of the true geometry by an infinite periodic beam. However, the quality of the band gaps remains, even for aperiodic structures.

\section{CONCLUSION}

The dynamic response of quasiperiodic beams resulting from two periodic sets of slits depends highly on the period ratio. A ratio $r=1$ leads to the well known case of a beam with periodic thickness variation, with multiple efficient wide band gaps but low overall stiffness due to the thin sections. An adiabatically varying beam with a ratio $r$ close to 1 behaves as a metawedge, trapping energy in the regions where a particular frequency is allowed to propagate. This leads to localized modes, and band-gap triggering depending on the 
location of the excitation point. Finally, beams with a larger period ratio exhibit interaction band gaps, a phononic effect that arises from linear combinations of the two main Brillouin zone edges. In this way, effective low-frequency band gaps can be achieved while maintaining a high bending stiffness, which is an important feat for applications.
[1] M. I. Hussein, M. J. Leamy, and M. Ruzzene, Appl. Mech. Rev. 66, 040802 (2014).

[2] B. R. Mace and E. Manconi, J. Sound Vib. 318, 884 (2008).

[3] Z. Liu, X. Zhang, Y. Mao, Y. Y. Zhu, Z. Yang, C. T. Chan, and P. Sheng, Science 289, 1734 (2000).

[4] M. Rupin, F. Lemoult, G. Lerosey, and P. Roux, Phys. Rev. Lett. 112, 234301 (2014).

[5] G. Lefebvre, A. Gondel, M. Dubois, M. Atlan, F. Feppon, A. Labbé, C. Gillot, A. Garelli, M. Ernoult, S. Mayboroda, M. Filoche, and P. Sebbah, Phys. Rev. Lett. 117, 074301 (2016).

[6] Y.-X. Xiao, Z.-Q. Zhang, and C. T. Chan, Sci Reports 8, 5160 (2018).

[7] S. H. Mousavi, A. B. Khanikaev, and Z. Wang, Nat. Commun. 6, 1 (2015).

[8] S. D. Huber, Nat. Phys. 12, 621 (2016).

[9] S. Yves, R. Fleury, T. Berthelot, M. Fink, F. Lemoult, and G. Lerosey, Nat. Commun. 8, 1 (2017).

[10] R. K. Pal and M. Ruzzene, New J. Phys. 19, 025001(2017).

[11] H. Abbaszadeh, A. Souslov, J. Paulose, H. Schomerus, and V. Vitelli, Phys. Rev. Lett. 119, 195502 (2017).

[12] M. Miniaci, R. K. Pal, R. Manna, and M. Ruzzene, Phys. Rev. B 100, 024304 (2019).

[13] A. Colombi, P. Roux, and M. Rupin, J. Acoust. Soc. Am. 136, EL192 (2014).

[14] A. Colombi, D. Colquitt, P. Roux, S. Guenneau, and R. V. Craster, Sci. Rep. 6, 1 (2016).

[15] R. K. Pal, M. I. N. Rosa, and M. Ruzzene, New J. Phys. 21, 093017 (2019).
[16] E. Riva, V. Casieri, F. Resta, and F. Braghin, Phys. Rev. B 102, 014305 (2020).

[17] J. M. Luck and D. Petritis, J. Stat. Phys. 42, 289 (1986).

[18] P. A. Kalugin, A. Bianchi, M. A. Chernikov, and H. R. Ott, Czech. J. Phys. 46, 2707 (1996).

[19] W. Steurer and D. Sutter-Widmer, J. Phys. D 40 (2007).

[20] A. L. Chen, Y. S. Wang, Y. F. Guo, and Z. D. Wang, Solid State Commun. 145, 103 (2008).

[21] M. I. N. Rosa, R. K. Pal, J. R. F. Arruda, and M. Ruzzene, Phys. Rev. Lett. 123, 034301 (2019).

[22] M. Gupta and M. Ruzzene, Crystals 10, 1144 (2020).

[23] S. Timorian, M. Ouisse, N. Bouhaddi, S. De Rosa, and F. Franco, Mech. Syst. Sig. Process. 136, 106516 (2020).

[24] W. Steurer and S. Deloudi, Acta Crystallogr., Sect. A 64, 1 (2008).

[25] D. Torrent, D. Mayou, and J. Sánchez-Dehesa, Phys. Rev. B 87, 115143 (2013).

[26] B. Van Damme and A. Zemp, Acta Acoust. United Acoust. 104 (2018).

[27] L. Liu and M. I. Hussein, J. Appl. Mech. 79, 011003 (2012).

[28] L. Ye, G. Cody, M. Zhou, P. Sheng, and A. N. Norris, Phys. Rev. Lett. 69, 3080 (1992).

[29] D. Gridin and R. V. Craster, Proc. R. Soc. London, Ser. A 460, 1831 (2004).

[30] A. Merkel, V. Romero-García, J.-P. Groby, J. Li, and J. Christensen, Phys. Rev. B 98, 201102(R) (2018).

[31] B. Van Damme and A. Zemp, Phys. Rev. Appl. 10, 014001 (2018). 